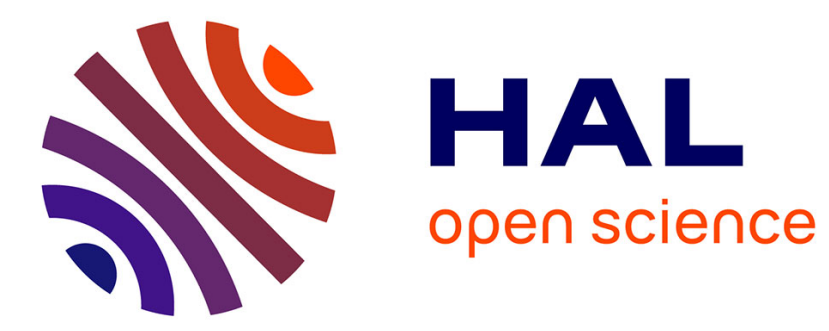

\title{
Giant Magneto impedance Sensor for Non Destructive Evaluation Eddy Current System
}

Rimond Hamia, Christophe Cordier, Sébastien Saez, Christophe Dolabdjian

\section{To cite this version:}

Rimond Hamia, Christophe Cordier, Sébastien Saez, Christophe Dolabdjian. Giant Magneto impedance Sensor for Non Destructive Evaluation Eddy Current System. Sensor letters, 2009, 7 (3, Sp. Iss. SI), pp.437-441. 10.1166/sl.2009.1045 . hal-00987150

\section{HAL Id: hal-00987150 https://hal.science/hal-00987150}

Submitted on 5 May 2014

HAL is a multi-disciplinary open access archive for the deposit and dissemination of scientific research documents, whether they are published or not. The documents may come from teaching and research institutions in France or abroad, or from public or private research centers.
L'archive ouverte pluridisciplinaire HAL, est destinée au dépôt et à la diffusion de documents scientifiques de niveau recherche, publiés ou non, émanant des établissements d'enseignement et de recherche français ou étrangers, des laboratoires publics ou privés. 


\title{
Giant Magneto Impedance Sensor for Non Destructive Evaluation Eddy Current System
}

\author{
R. Hamia*, C. Cordier, S. Saez, and C. Dolabdjian \\ GREYC (CNRS - UMR6072), ENSICAEN \& Université de Caen Basse Normandie, \\ 6 boulevard Maréchal Juin, 14050 Caen Cedex, France
}

\begin{abstract}
In this paper we present a simple and efficient Non Destructive Evaluation (NDE) eddy current system using GMI (Giant Magneto Impedance) magnetometer and single wire inducer. The performances of the system are determined and compared with a reference sensor. NDE measurements are done to detect corrosion defect on reference samples. A theoretical analysis with the finite element method compared to experimental measurements shows that the improved GMI (IGMI) magnetometer sensor could be easily used to detect defects like cracks or corrosions in agreement with sensor spatial resolution.
\end{abstract}

Keywords: Giant Magneto Impedance (GMI), Giant Magneto Resistance (GMR), Non Destructive Evaluation (NDE), Eddy-Current Testing (ECT), Finite Element Modeling (FE Method).

\section{INTRODUCTION}

Nondestructive inspection is widely employed to control the integrity of critical components in aerospace, automotive, nuclear and steel industries for example. Eddy current evaluation is a common method with applications ranging from crack detection to the sorting of material for their physical properties. These last years, the accuracy and reliability requirement of NDE have continuously increased. Probing local anomalies like cracks, corrosion, inclusions or others material defects, requires magnetic sensors having not only high field sensitivity, but also high field dynamic range, high spatial resolution and low intrinsic magnetic noise. The use of high sensitivity magnetic sensors has led to greatly improve non destructive evaluation eddy current systems. Their performances are now in competition with conventional detection sensors, like coil, in some practical cases. ${ }^{1}$ We present in this work a NDE system using high sensitivity magnetometer which has been developed in the electronic team of the GREYC research group. This magnetometer is made up of a GMI (Giant Magneto Impedance) wire as a sensitive element to the magnetic field.

The paper is organized as follows. The IGMI magnetometer and its performances are presented in Section 2. In Section 3, we briefly describe and underline the advantage

*Corresponding author; E-mail: rhamia@greyc.ensicaen.fr of an efficient finite element formulation to model the NDE system. Section 4 is devoted to the study of corrosion defects detection through experimentation and numerical simulation. This is then followed by a conclusion.

\section{NDE SYSTEM DESCRIPTION}

The NDE system used in this study is composed of a magnetometer which is built with a GMI element to sense the magnetic field. This element is a melt-extracted $\mathrm{CoFe}$ $\mathrm{SiBNb}$ soft amorphous magnetic wire from MXT Inc. of Montreal. ${ }^{2}$ Its electrical impedance is particularly sensitive to the component of an external magnetic field parallel to its main axis. The length of the wire used in this study is $18 \mathrm{~mm}$. The associated conditioning electronics consists in a simple RC oscillator, a diode-capacitance peak detector and an operational amplifier. More details on the electronics are presented in previous paper. ${ }^{3,4}$ The GMI radiofrequency bias current, provided by the oscillator, has a frequency of around $15 \mathrm{MHz}$ and amplitude of $20 \mathrm{~mA}_{\text {peak }}$. The highest maximal transfer values obtained with the GMI magnetometer is $90,000 \mathrm{~V} / \mathrm{T}$. In spite of its high sensitivity, this sensor exhibits a poor linearity and a high sensitivity variation. To get an efficient magnetometer for NDE measurements, by considerably improving the linearity and the dynamic range measurement of the sensor without degrading the sensor noise performance, we 


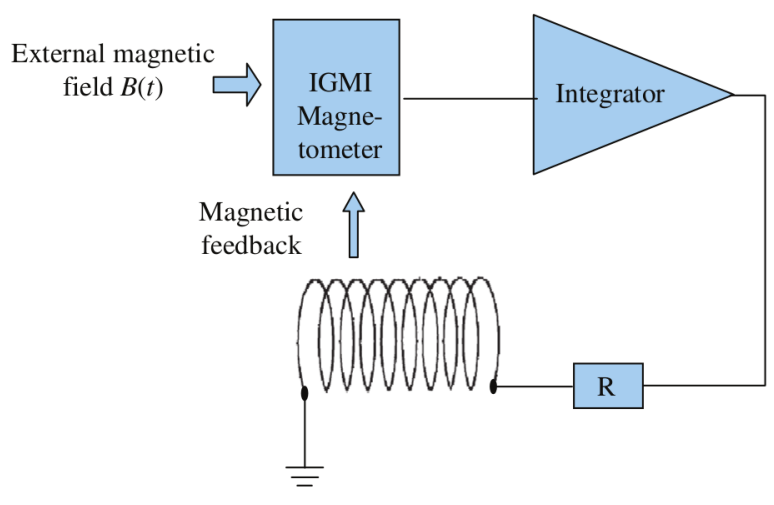

Fig. 1. Sketch view of improved GMI magnetometer using a very simple magnetic feedback loop system and a GMI sensor.

incorporate the GMI sensor in a magnetic feedback loop as summarized in Figure 1. The negative feedback field is applied to the GMI thanks to a coil wound around the wire. In that case, the system is able to be locked on an optimal working point with high sensitivity. The new IGMI magnetometer performances are summarized in Table I and compared to an IGMR magnetometer previously implemented and tested in an eddy current system. ${ }^{5}$

Both magnetometers have very good characteristics for eddy current NDE in shielded and unshielded environment. Theses sensors exhibit in particular a high field dynamic range. Although, most of their performances are comparable, the IGMI magnetometer is more sensible and less noisy than IGMR. Furthermore, the noise of the IGMI magnetometer is limited by the noise of the electronic device used for GMI sensor excitation and GMI sensor voltage detection. ${ }^{6}$ It means that higher performances could be obtained by reducing the noise of the associated electronic or by increasing the sensitivity of the sensor. Therefore, GMI sensors are promising for the development of magnetometers to improve classical eddy current magnetic inspection.

The full NDE system implemented is shown in Figure 2. It consists of an IGMI magnetometer and a single wire

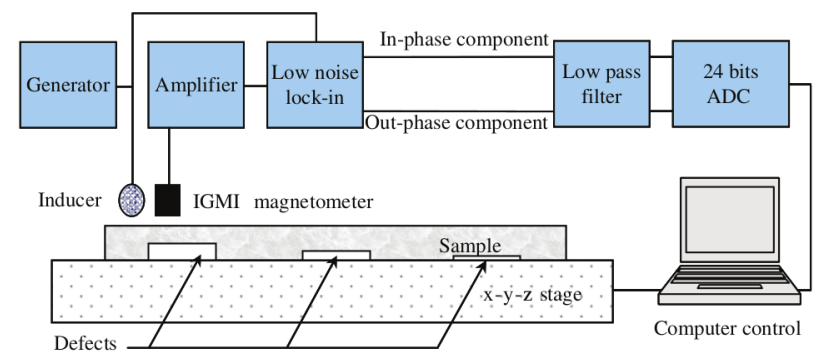

Fig. 2. Schematic diagram of the full NDE system.

inducer. A voltage generator provides a sinusoidal current through the inductor at the frequency $f_{0}$ and amplitude $I_{0}$. A lock-in amplifier gives the in-phase and out-phase voltage component corresponding to the magnetic field density detected by the IGMI magnetometer. These demodulated responses are low pass filtered and recorded with a PC by a 24 bits data acquisition and analysis software. The scanning of samples is experimentally done in our laboratory by a computer controlled $x-y-z$ stage.

The main advantage of the presented system is its modular aspect. Indeed, the simple wire inducer can be replace by another one, more suited to the targeted application. Furthermore numerous magnetometers can be easily added to form a sensor array, which is very convenient to inspect large zone or to control structure of complex surface with or without edges as previously given. ${ }^{5}$

\section{NUMERICAL MODELING OF THE NDE SYSTEM}

The NDE system used is given in Section 4. As an example, it permits to scan aluminum sample with typical defects. In order to support the analysis of experimental results, we have performed numerical simulations with COMSOL Multiphysics ${ }^{\circledR}$ software. ${ }^{7}$ The magnetic response of defects has been determined by using the finite element method. The first challenge of that kind of calculation comes from the high numerical precision required

Table I. Comparison of the improved GMI magnetometer performances to those of the improved GMR magnetometer.

\begin{tabular}{|c|c|c|}
\hline & Improved GMR magnetometer & Improved GMI magnetometer \\
\hline Spatial resolution & $\begin{array}{l}<<1 \mathrm{~mm} \\
\text { (limited by the encapsulation) }\end{array}$ & unmeasured \\
\hline Sensitivity & $\approx 1,000 \mathrm{~V} / \mathrm{T}$ & $\approx 90,000 \mathrm{~V} / \mathrm{T}$ \\
\hline Full Scale field range & $\pm 5 \mathrm{mT}$ & $\pm 100 \mu \mathrm{T}$ \\
\hline Bandwidth & $\mathrm{dc}$ to $>300 \mathrm{kHz}$ & dc to $>10 \mathrm{kHz}$ \\
\hline Slew-rate & $>37.5 \mathrm{~T} / \mathrm{s}^{*}$ & $>1.4 \mathrm{~T} / \mathrm{s}^{*}$ \\
\hline $\begin{array}{l}\text { Total Harmonic Distortion } \\
\qquad(\mathrm{f}=10 \mathrm{~Hz} \text { to } 1 \mathrm{kHz})\end{array}$ & $<0.03 \%{ }^{*}$ & unmeasured \\
\hline Noise density & $\begin{array}{l}3 / \sqrt{f} n T / \sqrt{H z} f<1 \mathrm{kHz} \\
\approx 100 p T / \sqrt{H z} f>1 \mathrm{kHz}\end{array}$ & $\begin{array}{l}630 / \sqrt{f} p T / \sqrt{H z} f<1 \mathrm{kHz} \\
\quad \approx 25 p T / \sqrt{H z} f>1 \mathrm{kHz}\end{array}$ \\
\hline Dynamic range ( $\mathrm{f}=10 \mathrm{~Hz}$ to $1 \mathrm{kHz}$ ) & 140 to $160 \mathrm{~dB} / \sqrt{\mathrm{Hz}}$ & 120 to $140 \mathrm{~dB} / \sqrt{H z}$ \\
\hline
\end{tabular}

${ }^{*}$ Results limited by benchmark. 
to get the variation of the signal induced by defects. High precision can be obtained by mesh refinement, but in the case of magnetic vector potential formulation, the memory required for calculation increase and rapidly exceeds the available computer RAM (6 GB). The second difficulty consists in scanning the sample by moving the inducer above the aluminum plate. Classically, the solution is to mesh the geometry for each position of the inducer and then assembly the matrices and solve the resulting numerical system. Nevertheless, this method leads to high CPU time calculation and often generates high numerical noise due to the mesh variability. Another advanced solution consists in using perturbation technique for the finite element method. ${ }^{8}$ The main advantage of this elegant method is to avoid remeshing. Perturbation approach for modeling nondestructive testing problem seems to be efficient especially for complex inducer including for example, high permeability flux concentrator. In the case of this study, the inducer is simply a wire connected to a sinusoidal current supply. Therefore, we have just chosen to implement in COMSOL Multiphysics ${ }^{\circledR}$ software, a classical electromagnetic formulation involving a magnetic vector potential associated to a magnetic scalar potential. More precisely, we have used the well-known edge finite element formulation $\boldsymbol{A}^{*} / \varphi_{r}$, in order to compute the magnetic flux density $\boldsymbol{B}$ on the whole space domain..$^{9,10}$ The modified magnetic vector potential $\boldsymbol{A}^{*}$ is only defined in the conducting region and is bound to the electric field $\boldsymbol{E}$ by

$$
\boldsymbol{E}=-\frac{\partial \boldsymbol{A}^{*}}{\partial t}=-\frac{\partial \boldsymbol{A}}{\partial t}-\nabla V
$$

where $\boldsymbol{A}$ and $V$ are respectively the magnetic vector potential and the scalar electric potential. The magnetic flux density in the conducting plate is obtained from

$$
\boldsymbol{B}=\nabla \times \boldsymbol{A}^{*}
$$

In the insulating region surrounding the aluminum sample, the magnetic field $\boldsymbol{H}$ is divided into two components

$$
\boldsymbol{H}=\boldsymbol{H}_{s}+\boldsymbol{H}_{r}
$$

Each part is determined by

$$
\begin{gathered}
\nabla \times \boldsymbol{H}_{s}=\boldsymbol{J}_{s} \\
\boldsymbol{H}_{r}=\nabla \varphi_{r}
\end{gathered}
$$

The source field $\boldsymbol{H}_{s}$ corresponds to the field created by the source current $\boldsymbol{J}_{s}$ in the inducer and is calculated by the Biot-Savart's law. The field $\boldsymbol{H}_{r}$ is the magnetic response of the conducting region to the source field. This component is derived from the reduced magnetic scalar potential $\varphi_{r}$. To calculate the potential $\boldsymbol{A}^{*}$ and $\varphi_{r}$, we have to consider the Maxwell-Ampere equation in the quasi-stationary state and the divergence free nature of the magnetic flux density. Finally to get the magnetic flux density we have to solve
Eq. (6) in region with eddy current and Eq. (7) in non conducting region, which gives, with the usual notations,

$$
\begin{gathered}
\nabla \times\left[\left(\mu_{0} \mu_{r}\right)^{-1} \nabla \times A^{*}\right]+j \omega \sigma A^{*}=0 \\
\nabla \cdot\left[\mu_{0} \nabla \varphi_{r}\right]=0
\end{gathered}
$$

The potentials $\boldsymbol{A}^{*}$ and $\varphi_{r}$ are coupled in the weak forms of Eqs. (6) and (7), thanks to the appropriate surface integrals over the boundary between the conducting and insulating region. Compared to the $\boldsymbol{A}-V$ formulation, the $\boldsymbol{A}^{*} / \varphi_{r}$ formulation has the advantage to bring a reduction of computing cost by decreasing the degrees of freedom in all the simulated regions. ${ }^{11}$ Furthermore, the scan of the sample is easily performed by changing a coordinate parameter in the expression of $\boldsymbol{H}_{s}$. With the use of a reduced potential, there is no need to discretize the inducer with finite elements, however the interface between the conductor and insulator must be discretized with care, especially if the inducer is close to the sample. Notice that this electromagnetic formulation works only for simply connected regions, but several technical solutions can be used to overcome this limitation. ${ }^{12,13}$

The numerical simulation of the sample studied in this paper was performed with the PARDISO solver on a dualcore processor at $2.13 \mathrm{GHz}$ (Intel Core 2 Duo E6400). The geometry of the problem was discretized with tetrahedral elements which led approximately to 210,000 degrees of freedom. The scan was done for 540 positions of the single wire inducer, with values well beyond the size of the aluminum sample. The CPU time needed for the solution was $1 \mathrm{~h} 07 \mathrm{~min}$, which is a good performance considering the number of positions computed and the required precision.

\section{EXPERIMENTAL AN THEORETICAL ANALYSIS}

We have used the improved GMI sensor to scan reference samples with artificial corrosion defects. Calibrated defects were machined into the subsurface of an aluminum plate which length, width and thickness are respectively $190 \mathrm{~mm}, 50 \mathrm{~mm}$ and $3 \mathrm{~mm}$. The geometry and positions of the defects are shown in Figure 3. Every defect differs from each other by their thickness which varies from $10 \%$ to $30 \%$ of the full sample thickness. The experimentations are done with current amplitude $I_{0}$ in the inducer set to $200 \mathrm{~mA}$. The excitation frequency $f_{0}$ is chosen equal to $1 \mathrm{kHz}$ so that the skin-depth in the aluminum plate $(\sigma=$ $1.3 \times 10^{7} \mathrm{Sm}^{-1}$ ) is equivalent to 1.47 times the plate thickness. The lift-off of the GMI magnetometer is set to $4 \mathrm{~mm}$, because of size restriction due to our experimental system. As it is shown on Figure 3, the GMI wire was positioned along the $x$ axis in order to measure the $x$ magnetic flux density component. Considering the $x$ component, instead of the other ones reduce by up to $40 \mathrm{~dB}$ the measured 


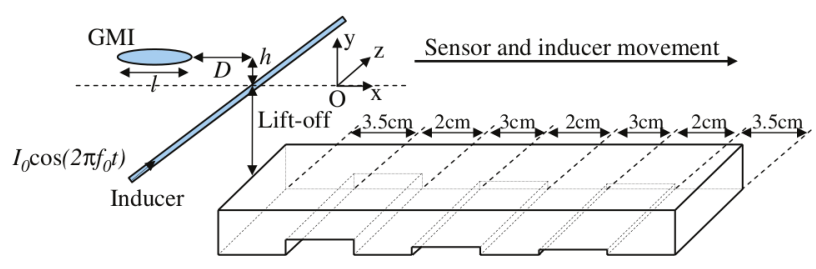

Fig. 3. The improved GMI magnetometer is associated to a single wire inducer to scan an aluminum plate with artificial corrosion defects. The length $l$ of the GMI wire is $18 \mathrm{~mm}$. The measurements were done for: a lift-off of $4 \mathrm{~mm}, D=1 \mathrm{~mm}$ and $h=2 \mathrm{~mm}$.

field when there is no sample under test. It means that the dynamic range of the magnetometer is mainly used for defects observations. ${ }^{14}$

The measurements of the magnetic response of the corrosion defects are reported in Figure 5. In a first step, we have compared the GMI response to the one given by an ideal punctual sensor set in the middle of the GMI wire. The magnetic flux density profiles are shown in Figures 5 and 6 and clearly state that, in that case, the IGMI magnetometer senses all defects. But, it cannot be considered as a punctual sensor. Afterwards, we have supposed that the GMI wire senses the mean magnetic flux density $\boldsymbol{B}_{x}$ observed along its length axis. With this simple hypothesis, each portion of the wire, which is considered to be connected in series to the near portion, has a local impedance variation which depends on the local magnetic field and which contributes to the global impedance response. By computing the integral of $\boldsymbol{B}_{x}$ along the $18 \mathrm{~mm}$ wire length we find results which are in a good agreement with experimental ones, as it is shown in Figure 5.

To confirm this hypothesis, the scan of the aluminum plate was also done by replacing the IGMI magnetometer with an IGMR magnetometer. In that case the spatial resolution of the sensor is less than $1 \mathrm{~mm}$ and the IGMR magnetometer response is compared with success to the response of an ideal punctual sensor in Figure 6.

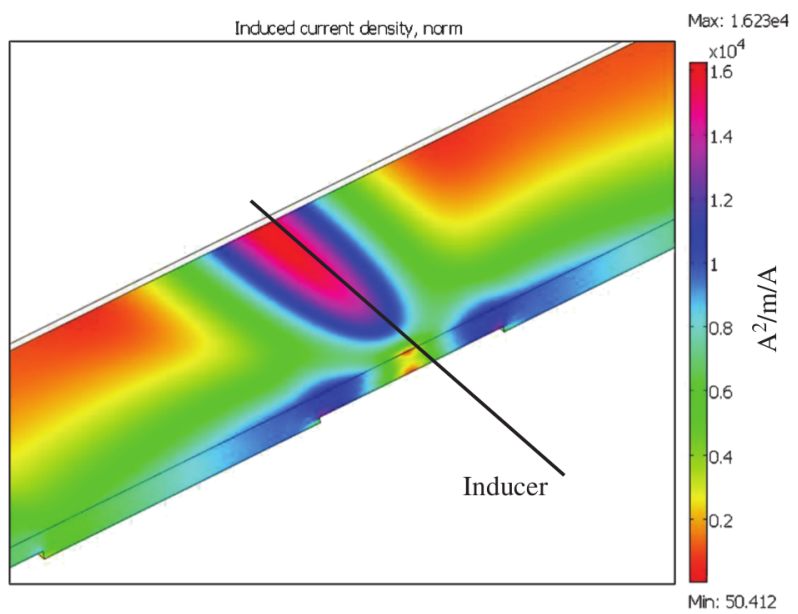

Fig. 4. Example of modulus induced current density image in a right half part of the sample (at inducer position of $x=0$, the plate center axis).

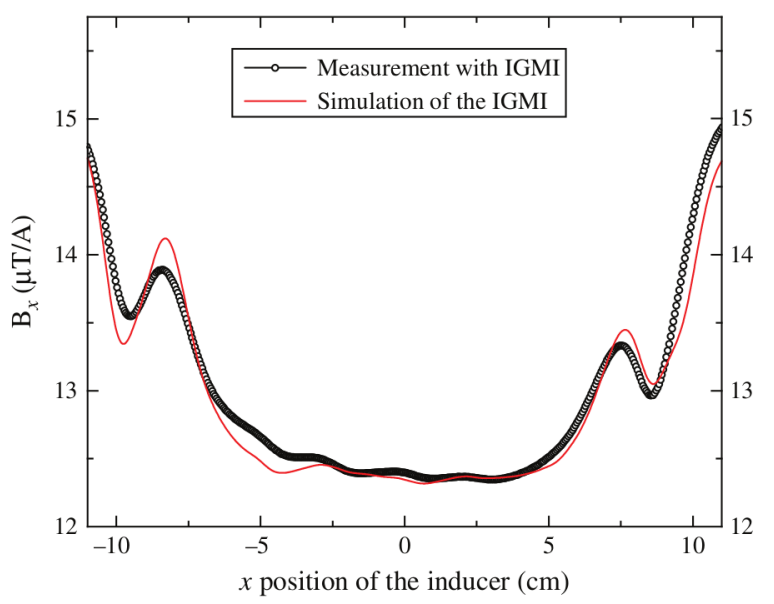

Fig. 5. Comparison of measured amplitude of $\mathbf{B}_{x}$ with the improved GMI magnetometer to the simulated one. $x$ represents the wire inducer position versus the plate center axis.

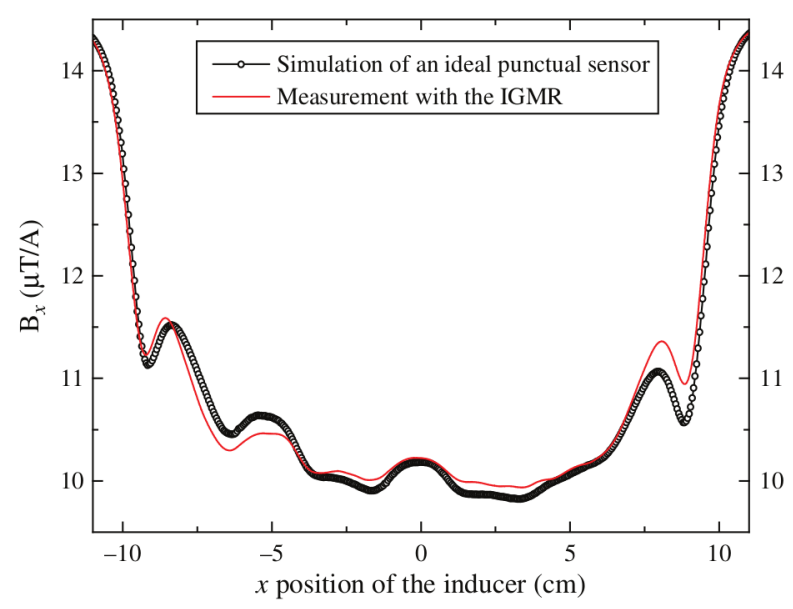

Fig. 6. Comparison of measured amplitude of $\mathbf{B}_{x}$ with the improved GMI magnetometer to the simulated one. $x$ represents the wire inducer position versus the plate center axis.

\section{CONCLUSION}

We have used COMSOL Multiphysics ${ }^{\circledR}$ software to model eddy current NDE scan of an aluminum sample with corrosion defects. The finite element formulation that we have implemented, leads to reasonable CPU times for the computation, and gives precise results with low numerical noise. In the case studied, we show that the IGMI magnetometer response depends mainly on the magnetic field component sensed along GMI wire length.

High sensitivity magnetometers such as improved GMR or improved GMI have a key place in the field of magnetic applications. Their measuring range and resolution placed these sensors between inexpensive Hall sensors and expensive SQUID or fluxgate magnetometers. The modular capabilities of these low cost probes and their high slew rate give them a high potential for NDE applications in harsh environment. Further works are in progress to improve, on one hand the GMI sensor sensitivity, and on 
the other hand the conditioning electronics to get a magnetometer with very low intrinsic equivalent magnetic noise density, closer to the intrinsic GMI noise density theoretically estimated well bellow the pT. ${ }^{6}$

\section{References and Notes}

1. L. Perez, J. Le Hir, C. Dolabdjian, and L. Butin, Journal of Electrical Engineering 55, 10 (2004).

2. MXT Inc., Montreal, QC Canada, http: //www.mxt.ca/

3. L. Ding, S. Saez, and C. Dolabdjian, Sensor Lett. 5, 248 (2007).

4. A. Boukhenoufa, C. Dolabdjian, and D. Robbes, IEEE Sens. J. 5, 916 (2005).

5. G. Waché, L. Butin, L. Perez, and C. Dolabdjian, Insight: The Journal of The British Institute of Non-Destructive Testing 47, 280 (2005).
6. L. Ding, S. Saez, C. Dolabdjian, L. G. C. Melo, A. Yelon, and D. Menard, IEEE Sens. J. (2008), in press.

7. COMSOL Multiphysics software, http: //www.comsol.com/

8. P. Dular and R.V. Sabariego, COMPEL International Journal for Computation and Mathematics in Electrical and Electronic Engineering 26, 700 (2007).

9. C. R. I. Emson and J. Simkin, IEEE Transaction on Magnetism 19 , 2450 (1983).

10. J. Simkin, IEEE Transaction on Magnetism 22, 609 (1986).

11. O. Biro and K. Preis, IEEE Transaction on Magnetism 25, 3145 (1989).

12. T. Morisue, IEEE Transaction on Magnetism 24, 110 (1988).

13. P. J. Leonard and D. Rodger, IEEE Transaction on Magnetism 24, 90 (1988)

14. L. Perez, C. Dolabdjian, W. Waché, and L. Butin, 16th World Conference on NDT, Montreal Canada (2004). 